\title{
Cosmopolitan freshwater bacteria in microbial communities of recreational freshwaters in Baikal region
}



\author{
Zaitseva S.V.*, Dagurova O.P., Tsyrenova D.D. \\ Institute of General and Experimental Biology, Siberian Branch of the Russian Academy of Sciences, 6 Sakhyanovoy str., Ulan-Ude, \\ 670047, Russia
}

\begin{abstract}
In the present study, three recreational freshwater lakes were analyzed covering a wide range of physical and chemical conditions resulting in distinct bacterioplankton community compositions. The bacterial diversity identified in three freshwater lakes further extends the presence and dominance of widely distributed few bacterial groups previously detected in freshwater lakes. The samples were quantitatively dominated by Bacteroidetes (13.7- 54.9\%), Gammaproteobacteria (17.7 - 59.8\%), Actinobacteria (6.7 - 27.9\%), Alphaproteobacteria (2.3 - 16.3\%), Cyanobacteria (0.6 - 30.3\%) and Verrucomicrobia $(2.4-6.5 \%)$, which is typical for freshwater environments. In our study, we examined the recovery of cosmopolitan freshwater bacterial groups according to physical and chemical properties of water in lakes. The distribution of some common freshwater taxa, which can act as indicator organisms and reflect any changes in the ecosystem caused by climatogenic and anthropogenic impact, was investigated. We observed the significant community distinction between the environments with high anthropogenic impact and samples without significant differences among three lakes environmental conditions.
\end{abstract}

Keywords: recreational freshwater, microbial community, environmental factor, high-throughput sequencing

\section{Introduction}

Recreational freshwaters provide important public services (Lee et al., 2016). Lakes are used for recreational purposes, such as swimming beach, picnic areas, and shelterhouses for many visitors. Anthropogenic impact on the water quality of recreational freshwaters can be well understood using various fecal indicating bacteria (Halliday et al., 2014). At the same time structural changes of microbial community can be also impacted by water quality parameters and environmental conditions in recreational freshwaters. The goal of this study was to elucidate whole microbial community by highthroughput sequencing and to evaluate relationships between the structure of microbial communities, water quality and environmental factors. With these data we also involve the distribution and growth traits of common freshwater taxa and identify the taxa responsible for differences in the microbial community depending on anthropogenic impact.

\section{Materials and methods}

Lakes Gusinoye $\left(164 \mathrm{~km}^{2}\right.$; maximum depth 26 $\mathrm{m})$, Shchuchye $\left(4.24 \mathrm{~km}^{2}\right.$; maximum depth $\left.23 \mathrm{~m}\right)$ and Kotokel $\left(68.9 \mathrm{~km}^{2}\right.$; maximum depth $\left.14 \mathrm{~m}\right)$ are large freshwater lakes located in Baikal region, Republic of
Buryatia (Russia). These lakes are used for household, fishery, drinking water supply and recreational purposes. The lakes are often influenced by non-point pollution sources from the surrounding watersheds, including croplands, livestock pasture, forestland, residential areas, and through recreational activities during summer. Lake Gusinoye is also impacted by anthropogenic activity from Gusinoozerskaya hydroelectric power station.

A total of 30 water samples were collected from three recreational freshwater lakes in August 2018. Water temperature, salinity (TDS), $\mathrm{pH}$, concentrations of nutrients and dissolved oxygen level were measured by portable instruments, cation and anion content, chlorophyll $a$ and total organic matter concentrations were determined in the laboratory in accordance with standard protocols.

Microbial diversity was studied by highthroughput sequencing using Illumina MiSeq platform in three technical replicates. The research was done using equipment of the Core Centrum 'GenomicTechnologies, Proteomics and Cell Biology' in ARRIAM.

\section{Results and Discussion}

Temperature in the sampling sites varied from 18.6 to $26^{\circ} \mathrm{C}, \mathrm{pH}-6.25$ to 8.61 . The concentration of

*Corresponding author.

E-mail address: svet_zait@mail.ru (S.V. Zaitseva)

(C) Author(s) 2020. This work is distributed under the Creative Commons Attribution 4.0 License. 
TDS in Kotokel (up to $145 \mathrm{mg} / \mathrm{L}$ ) tended to be less than those of the two other lakes (up to 281 and $381 \mathrm{mg} / \mathrm{L}$ ). The high nitrogen concentration and the highest total organic matter concentration were found in the sample of Kotokel Lake with cyanobacterial bloom. The highest sulfate concentration (up to $63 \mathrm{mg} / \mathrm{L}$ ) was determined in the surface water of Gusinoye Lake near household drain water.

At phylum level, three analyzed freshwater lakes presented similar microbial community structure with the dominance of few but typical freshwater bacterial groups even though conspicuous variations in their relative abundance were also observed. Taxonomic affiliations evidenced Bacteroidetes (range: 13.7\% - 54.9\%), Gammaproteobacteria (range: 17.7\% - 59.8\%), Actinobacteria (range: 6.7\% - 27.9\%), Alphaproteobacteria (range: $2.3 \%-16.3 \%$ ), Cyanobacteria (range: $0.6 \%$ - 30.3\%) and Verrucomicrobia (range: $2.4 \%-6.5 \%$ ) as six most abundant bacterial groups recovered in each lake (from first to sixth). The relative abundance of Planctomycetes-related OTUs was higher in Lake Gusinoe $(2.5 \pm 1.1 \%)$, and were less abundant in Lakes Shchuchie (1.0 $\pm 0.3 \%)$ and Kotokel $(0.8 \pm 0.5 \%)$. Cyanobacteria presented higher relative abundance $(30 \%)$ in the water sample of Lake Kotokel with high eutrophication and cyanobacterial bloom. Alphaproteobacteria were slightly more abundant in Lake Gusinoe (average for all sampling dates ca. 11.1\% of the total abundance) than in lakes Shchuchie (ca. 5.9\%) and Kotokel (ca. 4.1\%). Their greatest diversity (up to 53 OTU) was mainly represented by uncultivated bacteria. Considerable variations were observed in the bacterial community of Gammaproteobacteria, Alphaproteobacteria, Bacteroidetes, Actinobacteria and Cyanobacteria, where the bacterial community structure was greatly influenced by hydrological conditions and sample locations.

After examining the whole bacterial community composition differences between three lakes waters, we further examined the distribution of 15 common fresh water genera/lineages. The 15 genera/ lineages included: hgcl clade, Algoriphagus, Arcicella, Flavobacterium, Fluviicola, Sediminibacterium, SAR11, Sphingopyxis, Hydrogenophaga, Polynucleobacter, Pseudomonas, Acinetobacter, Rhodobacter, Rhodoferax and Luteolibacter. All genera/lineages were present in all samples $(\mathrm{n}=30)$ except for Sphingopyxis (29/30), Rhodobacter (24/30) and Arcicella (13/30). These 15 genera/lineages comprised on average 51.6 $\pm 4.5 \%$ of the sequence reads in the Lake Gusinoe water communities, on average $59.9 \pm 3.5 \%$ of the Lake Shchuchie and on average $55.6 \pm 15.2 \%$ of the Lake Kotokel communities. The minimum of these genera/lineages relative abundance (36-39\%) was founded in the sample Kotokel 3 of surface water with cyanobacterial bloom. The relative abundance of the entire cyanobacteria phylum was $30 \%$ in this sample and between $3 \%$ at other locations in Kotokel Lake. On the genus level, three major and 18 minor genera were identified. Among them Microcystis PCC7914 (17\% of relative abundance), Azolla filiculoides (7\%) and Aphanizomenon NIES81(5\%) were dominant.
Microcystis and Aphanizomenon are a toxic filamentous species that cause water blooms in freshwaters across the globe (Zhu et al., 2019). Harmful metabolites such as cyanobacterial toxins have increased public health risks (Valério et al., 2010; Zhu et al., 2019).

Previous studies showed that freshwater bacterial community structures have high genetic diversities and high spatiotemporal variation due to their sensitive response to dynamic conditions of aquatic environments (Newton et al., 2011; Newton and McLellan, 2015; Lee et al., 2016). Our results also revealed a number of interesting trends among the common freshwater genera/lineages. Specifically, members of the genus Flavobacterium and Rhodoferax exhibited high abundance correlations to high nutrient conditions in lakes associated with cyanobacterial bloom event or household drain water location.

\section{Conclusions}

Studies involving the distribution of common lake taxa have provided some insight into which taxa would be expected to drive changes across environmental gradients so that we could better resolve distribution patterns within some of the most common freshwater bacterial genera/lineages. Despite the near ubiquity of the 15 examined freshwater genera/lineages, we observed the significant community distinction between the environments with high anthropogenic impact and samples without significant differences among three lakes environmental conditions.

\section{Acknowledgments}

The work was supported by the Russian Foundation for Basic Research, project № 18-44030028 r_a, and partially within the framework of the State Assignment AAAA-A17-117011810034-9 for Institute of General and Experimental Biology, Siberian Branch, Russian Academy of Sciences.

\section{References}

Halliday E., McLellan S.L., Amaral-Zettler L.A. et al. 2014. Comparison of bacterial communities in sands and water at beaches with bacterial water quality violations. PLoSONE 9. DOI: $10.1371 /$ journal.pone.0090815

Lee C.S., Kim M., Lee C. et al. 2016. The microbiota of recreational freshwaters and the implications for environmental and public health. Frontiers in Microbiology 7. DOI: $10.3389 /$ fmicb. 2016.01826

Newton R.J., Jones S.E., Eiler A. et al. 2011. A guide to the natural history of freshwater lake bacteria. Microbiology and Molecular Biology Reviews 75: 14-49. DOI: 10.1128/ MMBR.00028-1

Newton R.J., McLellan S.L. 2015. A unique assemblage of cosmopolitan freshwater bacteria and higher community diversity differentiate an urbanized estuary from oligotrophic Lake Michigan. Frontiers in Microbiology 6. DOI: 10.3389/ fmicb.2015.01028

Valério E., Chaves S., Tenreiro R. 2010. Diversity and impact of prokaryotic toxins on aquatic environments: a review. Toxins 2(10): 2359-2410. DOI: 10.3390/ toxins 2102359 
Zhu B., Cao H., Li G. et al. 2019. Biodiversity and dynamics of cyanobacterial communities during blooms in temperate lake (Harsha Lake, Ohio, USA). Harmful Algae 82: 9-18. DOI: $10.1016 /$ j.hal.2018.12.006 